\title{
Understanding of Dry Eye in Subarachnoid Hemorrhage: An Experimental Study on the Role of Facial Nerve Ischemia
}

\author{
Osman TANRIVERDI', Mehmet Dumlu AYDIN² , Mehmet Resid ONEN ${ }^{3}$, Ilhan YILMAZ ${ }^{4}$, Mustafa KILIC ${ }^{4}$, \\ Nazan AYDIN ${ }^{5}$, Aslihan DUMAN ${ }^{6}$, Sevilay OZMEN ${ }^{7}$
}

\begin{abstract}
${ }^{1}$ University of Health Sciences, Bakirkoy Prof. Dr. Mazhar Osman Training and Research Hospital for Neurology, Neurosurgery and Psychiatry, Department of Neurosurgery, Istanbul, Turkey

${ }^{2}$ Ataturk University Medical Faculty, Department of Neurosurgery, Erzurum, Turkey

${ }^{3}$ University of Health Sciences, Umraniye Training and Research Hospital, Department of Neurosurgery, Istanbul, Turkey

${ }^{4}$ University of Health Sciences, Sisli Hamidiye Etfal Training and Research Hospital, Department of Neurosurgery, Istanbul, Turkey

EUniversity of Health Sciences, Bakirkoy Prof. Dr. Mazhar Osman Training and Research Hospital for Neurology, Neurosurgery and Psychiatry, Department of Psychiatry, Istanbul, Turkey

${ }^{6}$ Giresun University Medical Faculty, Department of Pathology, Giresun, Turkey

${ }^{7}$ Ataturk University Medical Faculty, Department of Pathology, Erzurum, Turkey
\end{abstract}

Corresponding author: Mehmet Dumlu AYDIN nmda11@hotmail.com

\section{ABSTRACT}

AIM: To understand possible mechanisms underlying lacrimal gland degeneration when facial nerve root ischemia induces pterygopalatine ganglion injury and subsequent dry eye in a rabbit model of subarachnoid hemorrhage.

MATERIAL and METHODS: Rabbits were divided into four groups: control, sham, moderate subarachnoid hemorrhage, and severe subarachnoid hemorrhage. Autologous blood recovered from the auricular artery was injected into the cisterna magna to induce subarachnoid hemorrhage in the two subarachnoid hemorrhage groups; animals were then monitored for dry eye development over 21 days before removal of their facial nerve roots, pterygopalatine ganglia, and lacrimal glands for immunohistochemical analyses. Neuronal viability in the pterygopalatine ganglia was measured; lacrimal gland vesicles were counted by stereological methods.

RESULTS: The mean tear-filled vesicle number and lacrimal gland volumes significantly decreased with an increase in facial nerve root injury severity and damaged neuron numbers in the pterygopalatine ganglion. Increase in injury severity most significantly decreased the tear-filled vesicle numbers in the pterygopalatine ganglion.

CONCLUSION: Subarachnoid hemorrhage degenerates facial nerve parasympathetic branches entering the pterygopalatine ganglion, and neuronal density in this ganglion may be correlated with tear secretion. Our data suggest that pterygopalatine ganglion degeneration following subarachnoid hemorrhage induces dry eye.

KEYWORDS: Dry eye, Subarachnoid hemorrhage, Lacrimal gland, Facial nerve, Pterygopalatine ganglion, Rabbit

\section{INTRODUCTION}

$\mathrm{T}$ The lacrimal gland (LG) produces most of the aqueous layer of the tear film, which contains water, electrolytes, and proteins for protecting and nourishing the ocular surface. Tear is produced by LG when irritation of the ocular surface activates afferent nerves of the cornea and conjunctiva, but chronic reductions in or lack of $L G$ secretion can cause the "dry eye" syndrome (31).

$L G$ is innervated by parasympathetic fibers in the facial nerve, which first synapse in the pterygopalatine ganglion
Osman TANRIVERDI (1D : 0000-0002-4846-4213

Mehmet Dumlu AYDIN (D) : 0000-0002-0383-9739

Mehmet Resid ONEN (1) : 0000-0003-4353-9068
Ilhan YILMAZ (D) : 0000-0001-5548-2228

Mustafa KILIC (D): 0000-0002-0701-5083

Nazan AYDIN (1) : 0000-0003-3232-7713
Aslihan DUMAN (10): 0000-0002-3629-0906

Sevilay OZMEN (1) : 0000-0002-1973-6101 
(PPG) before being carried as postsynaptic fibers into the orbitby zygomatic and zygomaticotemporal nerves (21). Immunofluorescence studies have shown that substance P, neuropeptide $\mathrm{Y}$, enkephalin and vasoactive intestinal peptide coexist in most neurons within the sublingual, submandibular, otic, and pterygopalatine ganglia as well as within the intracranial ganglion of the glossopharyngeal nerve (7). When describing the salivatory part of lacrimal nuclei and their intramedullary pathways, Martin and Mason stated that fibers from the greater superficial petrosal nerve enter the fasciculus solitarius and medial reticular formation (14).

A diverse set of sensory nerve fibers innervate the cornea. Stimulation of these fibers results in ocular dryness, discomfort, and pain (3). Sympathetic nerve fibers enter the orbit through the first and second trigeminal nerve (maxillary $-\mathrm{V}_{2}$ and mandibular $-\mathrm{V}_{3}$ - respectively) branches and form a nerve fiber network around the ophthalmic artery. Ocular structures are then innervated by sympathetic nerves via the posterior ciliary nerves. To innervate the sympathetic eyelid muscles, axons travel anteriorly in the orbit through the nasociliary and lacrimal nerves (26). The efferent arm of the corneal-LG reflex controls vasodilation in cat LGs by stimulating the corneal facial nerve root. This electrical stimulationis also correlated with evoked secretion in LG, suggestive of the types of autonomic ganglia and receptors contributing to lacrimal vasodilation and subsequent secretory responses (30). Surgical removal of the greater superficial petrosal nerve interrupts preganglionic parasympathetic neural control of $L G$ and results in dry eye (28). In addition, facial nerve palsy obstructs the functional nasolacrimal duct (2). Immunological ganglionopathies of PPG can also cause dry eye (24). Botulinum toxin can reduce tear volume and prevent corneal damage by increasing epidermal growth factor level without causing any histologic changes; thus, it is considered as a safe and effective treatment for patients with epiphora (12).

Subarachnoid hemorrhage (SAH) degenerates facial nerve parasympathetic branches entering PPG; PPG neuronal density may be correlated with tear secretion from LG. This indicates that PPG degeneration following SAH may lead to dry eye (Figure 1).

\section{MATERIAL and METHODS}

\section{Animal Selection and Study Groups}

Twenty-five rabbits were kept at room temperature in individual metal cages with a 12-hours light/dark cycle and $50 \%$ relative humidity. Food and water were provided ad libitum. Approval for all experiments was obtained from the ethics committee for animal experiments at Ataturk University School of Medicine.

The animals were randomly assigned to four groups: control, sham, and moderate SAH groups, each including five animals with silent clinical and histopathological findings, and a severe $\mathrm{SAH}$ group, including 10 animals. Following initial anesthesia with isoflurane, an anesthetic combination (ketamine HCL, 150 $\mathrm{mg} / 1.5 \mathrm{~mL}$; xylazine $\mathrm{HCl}, 30 \mathrm{mg} / 1.5 \mathrm{~mL}$; and distilled water, 1 $\mathrm{mL})$ was subcutaneously injected $(0.2 \mathrm{~mL} / \mathrm{kg})$ preoperatively; balanced, injectable anesthetics were used for reducing pain.
First, $1 \mathrm{~mL}$ autologous blood was recovered from the auricular artery; subsequently, the posterior notch of the foramen magnum was identified with the head in a hyper-flexed position and cerebrospinal fluid was aspirated by piercing the cisterna magna. Postoperatively, using a 22-gauge needle,1 $\mathrm{mL}$ autologous blood was injected into the cisterna magna of rabbits in the severe SAH group over approximately 1 minute. Rabbits in the sham group were injected with $1 \mathrm{~mL}$ serum saline through the same route. Rabbits in the control group underwent surgery but were not injected.

All animals were monitored for 21 days following surgery and injection without receiving medical treatment. Lacrimal secretion was determined by inserting a $10 \mathrm{~mm}$-diameter rolled cotton sphere into the lacrimal sac for approximately 4 min; the surface area absorbing secretions was then measured and used as the secretion score. Animals were sacrificed after 3 weeks; whole brains, facial nerves, and LGs were removed and stored in 10\% formalin solution for 7 days before histological examination. LGs, facial nerves, and PPGs were rehydrated through a graded series of ethanol and embedded in liquid paraffin for histological analysis.

\section{Histopathological Procedures}

LG was sliced into 5- $\mu \mathrm{m}$ sections at $30-\mu \mathrm{m}$ intervals; using the fractionator method, secretory follicle counting was performed

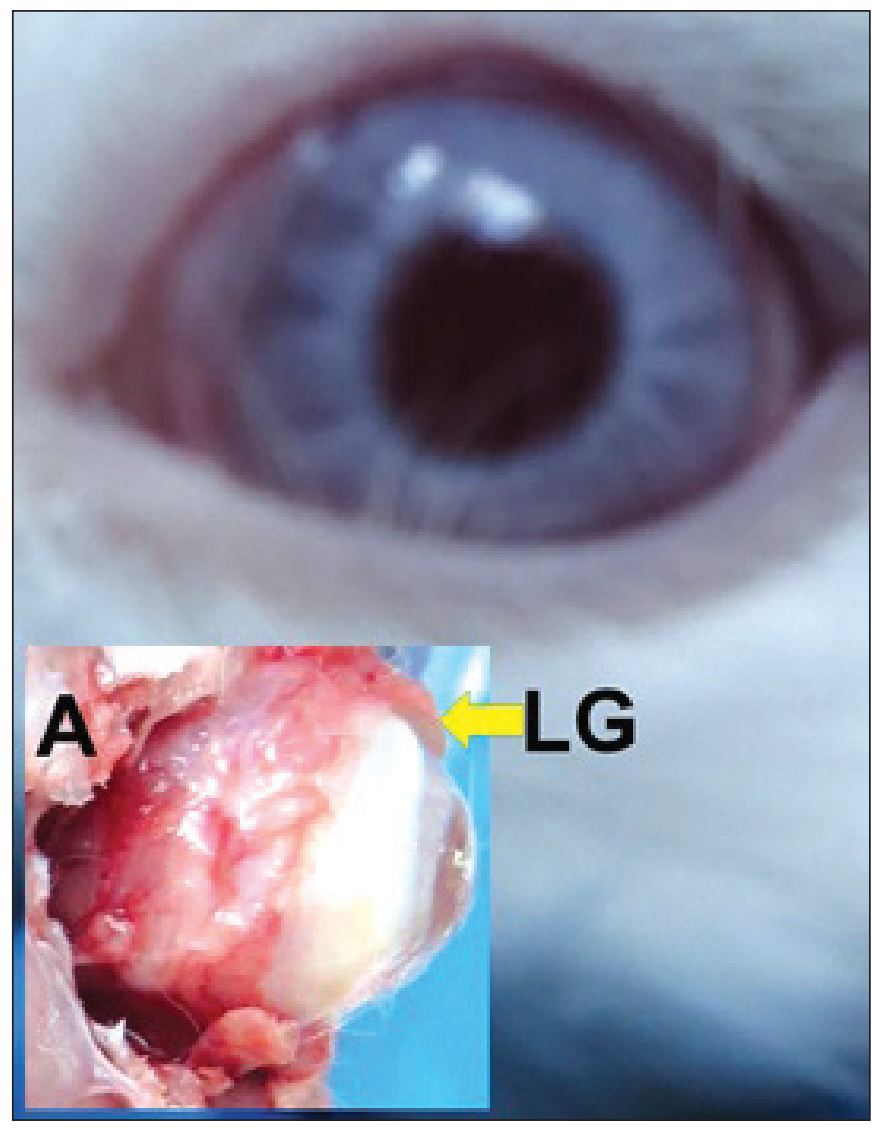

Figure 1: Macroscopic and anatomical appearance of dry eye (base) and the lacrimal gland at the superolateral part of the ocular bulb (LG/A), respectively, in a rabbit with $\mathrm{SAH}$. 
Tanriverdi O. et al: Dry Eye in Subarachnoid Hemorrhage

on the $30^{\text {th }}$ and $31^{\text {st }}$ sections. Tissue sections were placed on glass slides and stained with hematoxylin-eosin and TUNEL stain (Figures 2, 3).

Facial nerve lesions were analyzed using brain specimens sections that were parallel to the nerve long axis; both the axons and PPG were examined. Neuronal density was estimated in PPGs by longitudinally embedding glossopharyngeal nerve endings in paraffin blocks to observe the entire root system. Images of nerve root growth were then acquired using a Leica microscope (magnification, 20x and 40x). Lastly, neuronal

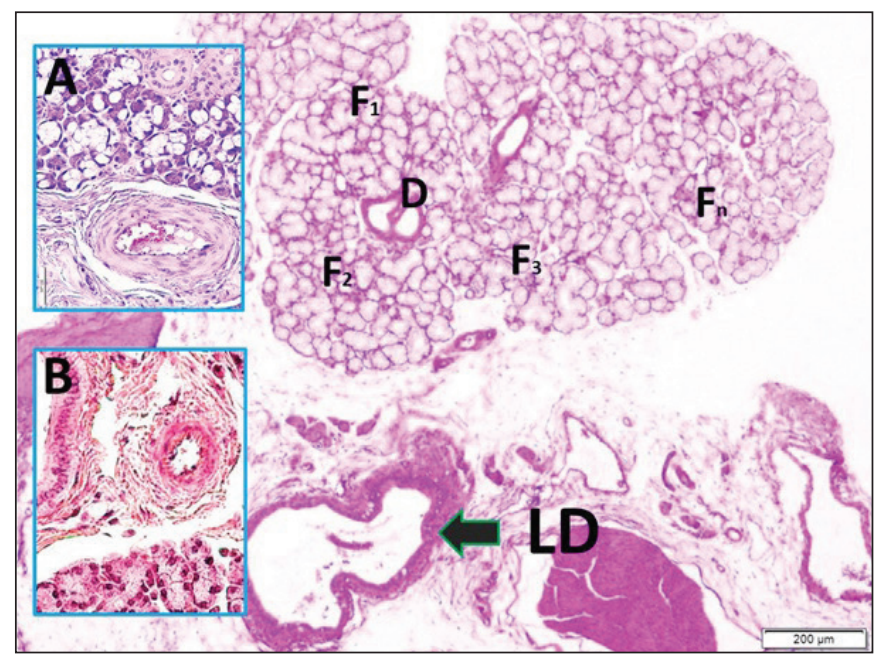

Figure 2: Histopathologic appearance of the lacrimal gland with tear follicles (Fn), ducts (D), and the lacrimal duct (LD) observed by light microscopy (LM) (H\&E, $\times 4 /$ Base); A: magnified image of tear vesicles (Fn) and the lacrimal arterial branch in a normal rabbit (LM, H\&E, $\times 4)$; and B: magnified image of the lacrimal gland with spastic arteries in a rabbit with severe SAH (LM, H\&E, $\times 10)$.

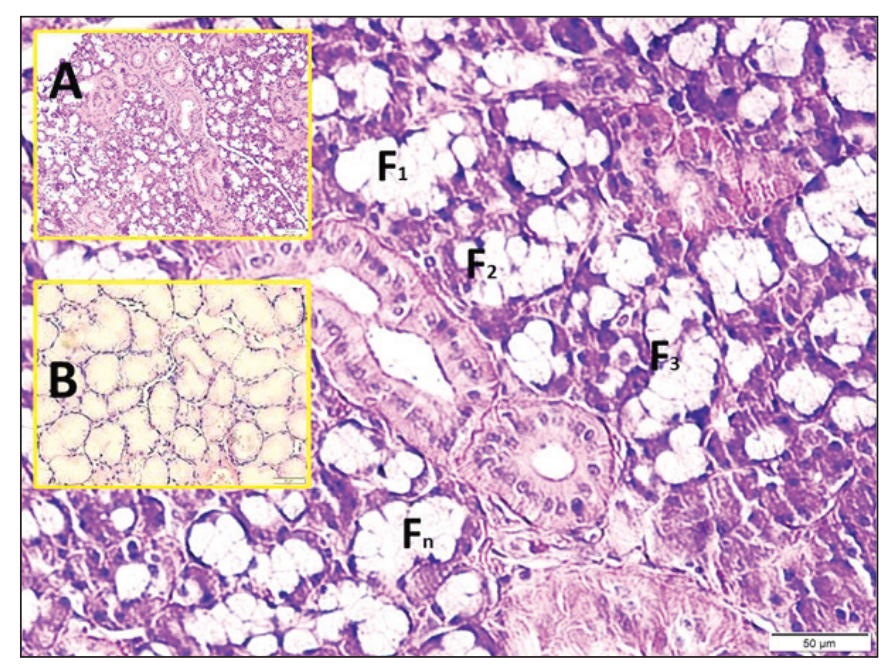

Figure 3: A: Histopathologic appearance of lacrimal gland tear follicles (Fn), ducts (D), and the lacrimal duct (LD) (LM, H\&E, x4); magnified images of tear vesicles (Fn) and duct branches in a normal rabbit (LM, H\&E, $\times 10 /$ Base); and B: magnified images of the lacrimal gland by Periodic acid and Schiff (PAS) staining in a normal rabbit (LM, PAS, $\times 10)$. density in each PPG was evaluated using the Cavalieri method (9).

\section{Stereological Analysis}

Stereological methods were used for measuring total respective structure volumes and total cell number and size (11). Consecutive sections were randomly selected from a starting point within the first 20 -section interval. There after, every $20^{\text {th }}$ and $21^{\text {st }}$ sections were sampled, giving a section sampling fraction ( $f 1$ ) of $1 / 30$. Section pairs that did not contain the parotids and ganglia were discarded. Such a sampling fraction produced an average of $10-11$ section pairs, with a sampling fraction (f2) area of 1/1. The lacrimal saliva follicle number was evaluated using the physical dissector method. The method easily estimates particle number; is intuitive and easy to perform; does not assume the particle size, shape, and orientation; and is not affected by over protection or truncation. Two consecutive sections (dissector pairs) obtained from tissue samples with named reference sections were mounted on each slide. The dissector pair number was doubled without obtaining more sections by swapping reference and look-up sections. The following equation was used for estimate the total saliva-filled follicle number $(N)$ in $L G$, with $\Sigma Q^{-}$denoting the follicle count:

$N=\sum Q^{-} \times \frac{1}{f_{1}} \times \frac{1}{f_{2}}$

The same method was used to evaluate healthy and damaged neuron numbers in PPG. Neuronal angulation, cytoplasmic condensation, nuclear shrinkage, or increased apoptosis were considered to indicate neuronal damage. Two consecutive sections (dissector pairs) were placed on each slide and the paired reference sections were reversed (Figure 4A, B). The following formula was used for estimating the mean PPG neuronal density ( $\mathrm{Nv} / \mathrm{Gv}$, per $\mathrm{mm}^{3}$ ) (16), where $\Sigma Q^{-}$is the total neuron count observed in the reference section only, $d$ is section thickness, and $A$ is the frame area:

$$
N_{v} / G_{V}=\sum Q^{-} / \sum A \times d
$$

The $\Sigma$ A might be effectively estimated for a set of dissectors using, where $\Sigma \mathrm{P}$ is the total counting set frame point number and $a$ is the constant area associated with the set point. The total neuron number in each sample was estimated using the Cavalieri method; multiplying neuronal density in each PPG with the volume $\left(\mathrm{mm}^{3}\right)$ provided the total neuron count. Subsequently, differences between salivary follicle numbers and degenerated neuron densities in PPG were compared.

\section{Statistical Analyses}

Experimental data were analyzed using SPSS for Windows v. 12.0 (SPSS Inc., Chicago, USA). Data of the four groups were compared using the Mann-Whitney $U$ test for pair-wise comparisons of independent samples and were subsequently analyzed using the Kruskal-Wallis test. p-values for multiple comparisons were calculated by dividing 0.05 by using Bonferroni correction and were considered to be statistically significant when $\leq 0.0034$. Results were considered statistically significant for $\mathrm{p}$ values $<0.05$. 
Tanriverdi O. et al: Dry Eye in Subarachnoid Hemorrhage

The vasospasm index (VSI) was calculated by dividing the proportion of external surface with the lumen surface as follows: $\left(\pi R^{2}-\pi r^{2} / \pi r^{2}=\left(R^{2}-r^{2}\right) / r^{2}\right.$ (lacrimal artery: external diameter, 2R; internal diameter:2r). After counting, the total vesicle volumes (TVV) were estimated by adding vesicle volumes. Each vesicle was considered to be spherical; their volumes were calculated using the following formula: $\mathrm{Vn}=$ $4 / 3 \pi r_{n}{ }^{3}$. TVV was estimated using the following formula: TVV $=\mathrm{n} \times \mathrm{Vn}$.

\section{RESULTS}

Three animals in the intervention groups and one animal in the sham group died within 7 days postoperatively and were replaced. Fever, convulsive attacks, unconsciousness, breathing disturbances, apnea, cardiac arrhythmia, and neck stiffness were observed in all hyperthermic animals. During the initial phase of fatal $\mathrm{SAH}$, the average heart rate increased to $361 \pm 46 / \mathrm{min}$ and respiration frequency decreased to 13 $\pm 3 /$ min, with increased respiratory amplitudes. However, this progressed to tachypnoea and decreased amplitudes, leading to shortened inspiration, longer expiration, apnea-tachypnoea attacks, diaphragmatic breathing, and ultimately, respiratory arrest. Massive SAHs were also observed in basal cisterns of animals in the G IIIA-B group, resulting in meningeal irritation and cardiorespiratory dysrhythmia.

In control animals, the average heart rate, respiratory rate, and blood oxygen concentration were $265 \pm 14$ /minutes, $32 \pm 6$ / minutes, and $94 \% \pm 6 \%$, respectively, but shortly after $\mathrm{SAH}$, they decreased to $145 \pm 27 /$ minutes, $17 \pm 4 /$ minutes, and $75 \%$ $\pm 8 \%$, respectively. Significant electrocardiographic changes, including ventricular extrasystoles, bigeminal/trigeminal extra systoles, ST depression, QRS separation, and fibrillations, were also observed. The mean normal neuronal density in PPG of all animals was $11.156 \pm 982$ per $\mathrm{mm}^{3}$, where as the mean damaged neuronal density was $17 \pm 3,45 \pm 9,1.230 \pm$ 98 , and $3.127 \pm 763$ per $\mathrm{mm}^{3}$ in the control, sham, moderate $\mathrm{SAH}$, and severe SAH groups, respectively.

Corneal moisture levels were examined using three-piece napkins (surface area, $4 \mathrm{~mm}^{2}$; height, $0.5 \mathrm{~mm}$ ) five times a day. A total of $12-15,8-12$, and $<5$ wet napkin pieces/day were considered to be normal, below normal, and dry eye, respectively. Following $\mathrm{SAH}$, mean tear-filled vesicle numbers and LG volumes differed with facial nerve root injury severity and damaged neuron density in PPG (Figure 5). The mean tear vesicle numbers and damaged neuron density in PPG in the control, sham, moderate $\mathrm{SAH}$, and severe SAH groups were $3.12 \pm 0.94$ and $23.43 \pm 5.13,2.83 \pm 0.75$ and $64.46 \pm 11.24$, $2.05 \pm 0.45$ and $143.27 \pm 32.41$, and $1.56 \pm 0.22$ and $478.78 \pm$ 102.34 per $\mathrm{mm}^{3}$, respectively.

The total tear-filled vesicle number in LGs in each group was also stereologically assessed: $643.65 \pm 95.76$ in the control group, $561.54 \pm 87.09$ in the sham group, $434.87 \pm 64.23$ in the moderate $\mathrm{SAH}$ group, and $341.46 \pm 75.56$ in the severe $\mathrm{SAH}$ group (Table I). Overall, tear-filled vesicle numbers significantly decreased with increase in SAH-related damage $(p<0.05)$.

\section{DISCUSSION}

Parasympathetic fibers from facial nerves innervate LGs; if this network is damaged, the production of tears decreases and causes dry eye. One cause is $\mathrm{SAH}$-induced denervation
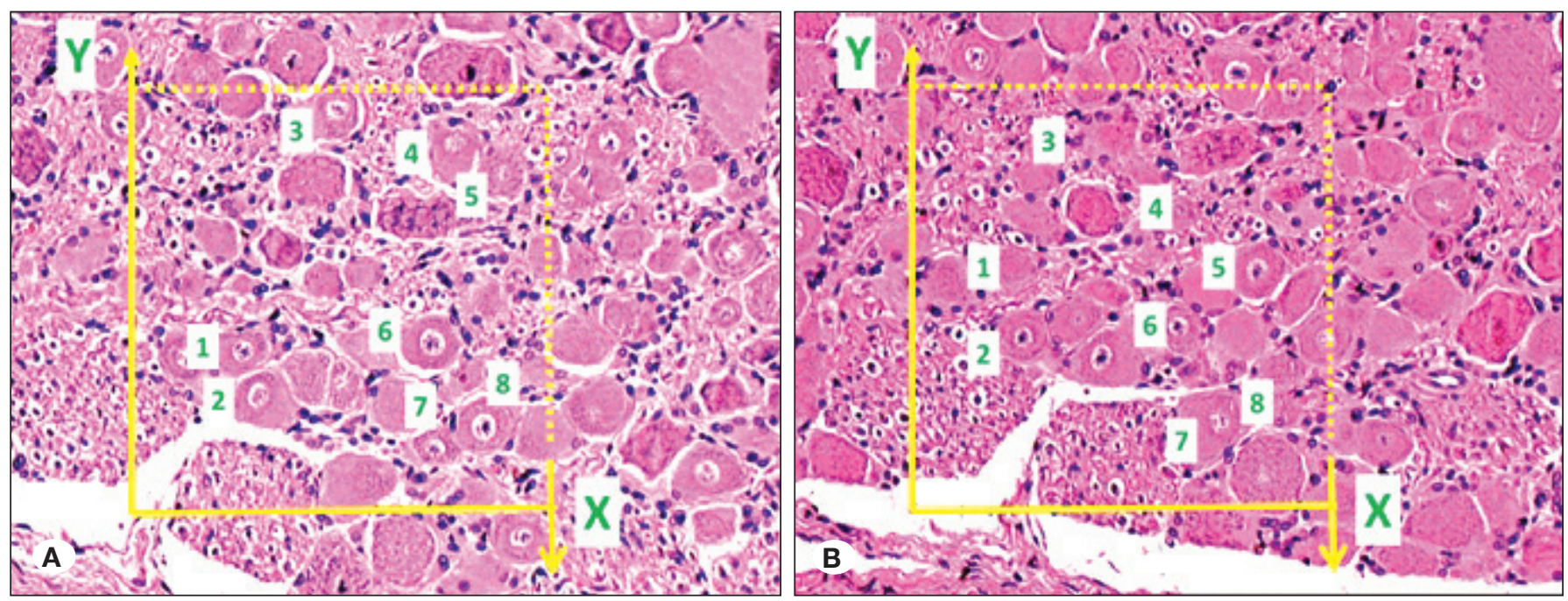

Figure 4A, B: Stereological cell counting of the pterygopalatine ganglion.

Application of the physical dissector method in which micrographs in the same fields of view (A, B) were taken from two parallel, adjacent, thin sections separated by $5 \mu \mathrm{m}$. The upper and right lines in the unbiased counting frames represent inclusion lines; the lower and left lines, including the extensions, represent exclusion lines. The neuronal nucleoli touching the inclusion lines were excluded; nucleoli profiles touching the inclusion lines and located inside the frame were counted as dissector particles unless their profile extended up to the reference section. Number of neurons from the two dissectors in a volume was found by the counting frame area and distance between sections. The nucleoli marked 1, 3, 4, and 8 are dissector particles because they are in section A but not section B. However, the nucleoli marked 2, 3, 5, 6, and 7 are not dissector particles because they are present in both sections (LM, H\&E, $\times 10)$. 
injury of the parasympathetic ganglia. Here we used a rabbit model of SAH to improve our understanding of mechanisms underlying $L G$ degeneration related to PPG injury induced by facial nerve root ischemia, and consequently leading to dry eye.

Neuronal density in PPG may be important for tear secretion from LG; SAH degenerates facial nerve parasympathetic branches entering PPG. These data suggest that PPG degeneration induces dry eye following SAH. LG tears comprise three layers, with different tissues or glands responsible for each layer: together, LG, efferent nerves of LG, and afferent nerves of the cornea and conjunctiva work as a functional unit for producing aqueous tear components $(4,20)$. In addition to water and electrolytes, LG secretes the major protein component of tears, helping protect and nourish the ocular surface (5). Tears are secreted in response to environmental stress that is potentially harmful to the ocular surface, including infections, injury, mechanical or noxious stimuli, and cold (10).

We aimed to investigate the interaction between tear secretion and corneal stimulation at different positions to determine the possible relationship between detection thresholds for

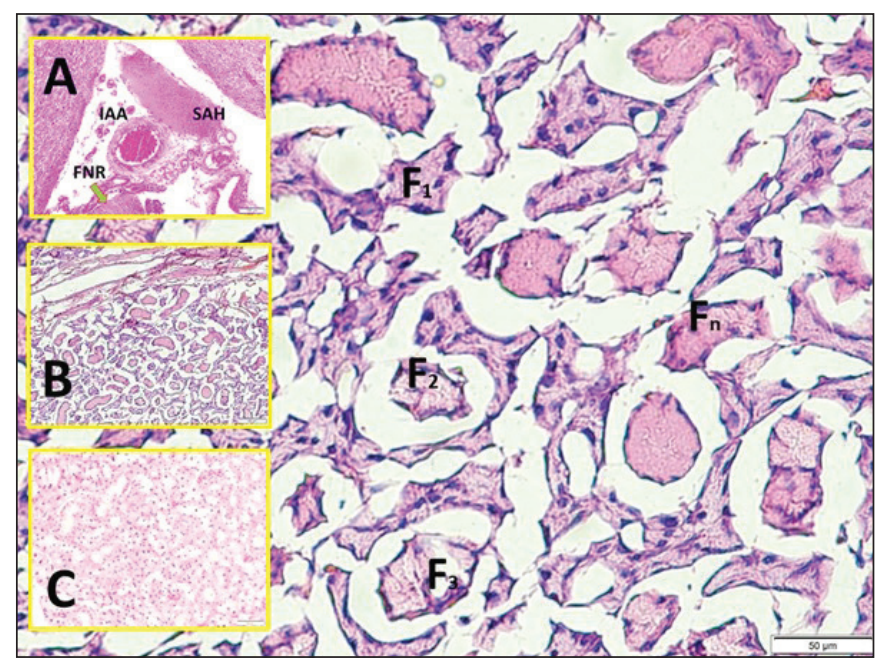

Figure 5: A: Histopathological appearances of the pontocerebellar cistern filled with SAH, facial nerve roots (FNR), and internal auditory artery (IAA) (LM, H\&E, $\times 4)$ B: as well as the lacrimal gland with atrophic follicles in rabbit with SAH (LM, H\&E, x4); C: magnified form of $B$ and a decrease in the folliclesize in $L G$ is visible (LM, PAS, $\times 4)$. nociceptive stimuli and tear production. We found that the strongest lacrimation reflex was evoked by mechanical stimulation applied to the central cornea, and considerable tear secretion was induced by noxious chemical and mechanical stimuli. Mechanical stimulation to the central cornea is the most effective combination of stimulus and position for tear secretion by an LG reflex (23). We also found that PPG degeneration following SAH might be an important factor in dry eye development.

The study on human nasolacrimal ducts and associated disorders is at an intersection of ophthalmology and otorhinolaryn- gology. However, knowledge on the physiology and pathophysiology of the nasolacrimal system is limited. The system comprises the lacrimal sac; nasolacrimal duct; and upper and lower lacrimal canaliculi, its primary purpose being channeling tears from the ocular surface to the lower meatus of the nose. The hypothesis on the absorption of tear fluid components in the nasolacrimal system in humansis supported by animal experiments conducted using rabbits. The presence of seromucous glands, intra-epithelial lipids, and a specific mucus layer in the nasolacrimal ducts supports the faction of all three layers in producing the tear film. Goblet cells and intra-epithelial mucus glands synthesize the mucus layer, which is a drainage mechanism for tear fluid, that has properties similar to those of the gastrointestinal tract epithelia. Antimicrobial peptide (e.g., IgA) secretion and intra-epithelial and sub-epithelial immunocompetent cells (e.g., lymphocytes and macrophages) presence indicate involvement of this tissue in local defense (17).

Neural control through a reflex arc is the primary mechanism for controlling LG secretion. Afferent nerves of the cornea and conjunctiva are activated by ocular surface stimulation. This, in turn, activates efferent parasympathetic and sympathetic nerves in $L G$ to stimulate secretion. For example, unilateral sympathetic nerve stimulation results in approximately $60 \% \mathrm{LG}$ blood flow reduction (8). Moreover, LG function is regulated by steroid sex hormones (31). The presence of vasoactive intestinal polypeptide-like proteins in facial parasympathetic nerves innervating the eye and LG suggests that this peptideis an important co-transmitter or neuromodulator in these nerves (22).

Understanding age-dependent changes in the functional capacity of $L G$ are equally important for understanding $L G$ function and potential treatments for dry eye. Although various mechanisms have been proposed, detailed mechanisms

Table I: The Total Tear-Filled Vesicle Number In Lacrimal Glands

\begin{tabular}{lcccc}
\hline & Control Group & SHAM & $\begin{array}{c}\text { SAH group I } \\
\text { Slight neuron density }\end{array}$ & $\begin{array}{c}\text { SAH group II } \\
\text { Severe neuron } \\
\text { density }\end{array}$ \\
\hline Tear vesicles & $3.12 \pm 0.94$ & $2.83 \pm 0.75$ & $2.05 \pm 0.45$ & $1.56 \pm 0.22$ \\
\hline Damaged neurons in the PPG & $23.43 \pm 5.13 / \mathrm{mm}^{3}$ & $64.46 \pm 11.24 / \mathrm{mm}^{3}$ & $143.27 \pm 32.41 / \mathrm{mm}^{3}$ & $478.78 \pm 102.34 / \mathrm{mm}^{3}$ \\
\hline $\begin{array}{l}\text { Total number of tear filled } \\
\text { vesicles in the lacrimal gland }\end{array}$ & $643.65 \pm 95.76$ & $561.54 \pm 87.09$ & $434.87 \pm 64.23$ & $341.46 \pm 75.56$ \\
\hline
\end{tabular}


of age-related factors affecting LG remain unclear. Proposed mechanisms are related to the program theory, which proposes the involvement of internal factors (i.e., genetic and neuroendocrine), or the damage theory, which proposes the involvement of external factors (i.e., mutation and protein alteration). It is also possible that hyperglycemia and lymphocytic infiltration are involved in structural and functional changes and the loss of function observed in LG with age. The age-related loss of LG function may itself originate from the increased oxidative stress associated with age (20).

Dry eye syndrome may be an inflammatory disease of the ocular surface and LG. Inflammation of LG is common in several pathologies and may be due to aging, autoimmune diseases (e.g., Sjögren's syndrome), or host reactions to organ transplantation. Dry eye also results from facial nerve diseases, with immunological ganglionopathies of PPG being the primary cause $(13,24)$, as well as autoimmune dacryoadenitis (27). Main indicators of LG inflammation are focal lymphocytic infiltrates and proinflammatory cytokines in LG; however, their role in mechanisms underlying $L G$ dysfunction remains poorly understood. Possible mechanisms underlying LG dysfunction are neural dysfunction, altered signaling, hormonal imbalance, autoantibodies, proinflammatory cytokines, and apoptosis $(1,6,31)$.

Tear production from LG is stimulated through facial nerve parasympathetic fibers. After their first synapse in PPG, postsynaptic fibers are carried into the orbitby the zygomatic and zygomaticotemporal nerves. Before secretomotor fibers connect to the gland, the zygomaticotemporal nerves link with LG behind the gland $(21,29)$. The salivatory nuclei is usually located between levels of the facial nerve root and the rostral tip of the nucleus of the solitary tract in rat brain maps; most studies have focused on the superior salivatory nucleus. The association between the lacrimal and submandibular subnuclei and between the lacrimal and inferior subnuclei of the superior salivatory nucleus has been defined by Rezek et al., who stated that lacrimal subnucleus was located ventrolateral to the caudal portion of the inferior subnucleus (18). Accordingly, we suggest that facial nerve injury causes dry eye and mouth. Facial nerve palsy causes functional nasolacrimal duct obstruction and may lead to pathological lacrimation (2). Injecting botulinum toxin into LG isa reported suitable treatment option (19). The pterygopalatine parasympathetic nerves innervate LGs. Although direct synaptic contacts were not found in the current study, several unmyelinated fibers and vesicle-containing terminals were observed in these structures. Similar findings were obtained in previous studies on cats and monkeys (2).

In rats, PPG comprises a triangular portion and supplemental perikarya which encircled the distal part of the major petrosal nerve. Through the inferior petrosal sinus, the superior cervical ganglion fibers stretch to PPG. A previous histochemistry and autoradiography study labeled fibers in PPG with rostral application to PPG and the preganglionic parasympathetic brainstem neurons dorsolateral to the facial nucleus (i.e., the lacrimal nucleus) as well as rostral cells in the superior cervical ganglion and trigeminal sensory fibers with caudal application to PPG. Thus, it was suggested that PPG includes afferent and sympathetic nerves and has a topographical organization along the rostrocaudal axis. Fibers passing through the ciliary ganglion, which innervates the conjunctiva, limbus, and parts of the choroid, were labeled by isotope injections to PPG in an anterograde fashion (25).

Surgical removal of the greater superficial petrosal nerve causes a rapid onset of dry eye that lasts for more than a week. Thus, constant PPG stimulation may be needed for sufficient tears and mucin secretion, and the trigeminal nerves may be the afferent nerves responsible for this continuous neural tone (28). Major structural changes also occur in LG when muscarinic parasympathetic input is lost, with a significant concomitant tear secretion reduction. On study has shown that when the greater superficial petrosal nerve was severed (i.e., the input to PPG), gene expression was altered. This negatively affected the secretory pathway and upregulated pathways for cell proliferation and inflammation, suggesting a loss of organization in LG after parasympathetic input removal (15).

\section{CONCLUSION}

Dryness of the mouth, eyes, paranasal sinus, and upper respiratory tracts observed after SAH are known to be related to hyperpyrexia. However, in this study, we showed that the pathological mechanism of dry eye was also associated with the subarachnoid part of the facial nerve. Specifically, PPG degeneration caused significant loss in tear production because of lack of LG innervation.

\section{口 REFERENCES}

1. Baudouin C: A new approach for better comprehension of diseases of the ocular surface. J Fr Ophtalmol 30:239-246, 2007

2. Becker BB: Secondary dye testing of the lacrimal system. Ophthalmic Surg 24:91-93, 1993

3. Belmonte C: Eye dryness sensations after refractive surgery: Impaired tear secretion or "phantom" cornea. J Refract Surg 23:598-602, 2007

4. Dartt DA: Dysfunctional neural regulation of lacrimal gland secretion and its role in the pathogenesis of dry eye syndromes. Ocul Surf 2:76-91, 2004

5. Evans E, Zhang W, Jerdeva G, Chen CY, Chen X, HammAlvarez SF, Okamoto CT: Direct interaction between Rab3D and the polymeric immunoglobulin receptor and trafficking through regulated secretory vesicles in lacrimal gland acinar cells. Am J Physiol Cell Physiol 294:662-674, 2008

6. Fox RI, Maruyama T: Pathogenesis and treatment of Sjögren's syndrome. Curr Opin Rheumatol 9:393-399, 1997

7. Gibbins IL: Target-related patterns of co-existence of neuropeptide $\mathrm{Y}$, vasoactive intestinal peptide, enkephalin and substance $P$ in cranial parasympathetic neurons innervating the facial skin and exocrine glands of guinea-pigs. Neuroscience 38:541-560, 1990 
8. Granstam E, Nilsson SF: Non-adrenergic sympathetic vasoconstriction in the eye and some other facial tissues in the rabbit. Eur J Pharmacol 175:175-186, 1990

9. Gundersen HJ, Bendtsen TF, Korbo L, Marcussen N, Møller A, Nielsen K, Nyengaard JR, Pakkenberg B, Sørensen FB, Vesterby A, West MJ: Some new, simple and efficient stereological methods and their use in pathological research and diagnosis. APMIS 96:379-394, 1998

10. Hodges RR, Dartt DA: Regulatory pathways in lacrimal gland epithelium. Int Rev Cytol 231:129-196, 2003

11. Kanat A, Turkmenoglu O, Aydin MD, Yolas C, Aydin N, Gursan N, Tumkaya L, Demir R: Toward changing of the pathophysiologic basis of acute hydrocephalus after subarachnoid hemorrhage: A preliminary experimental study. World neurosurgery 80:390-395, 2013

12. Kim JW, Baek S: Functional and histologic changes in the lacrimal gland after botulinum toxin injection. J Craniofac Surg 24:1960-1969, 2013

13. Li N, Deng X, Gao Y, Zhang S, He M, Zhao D: Establishment of the mild, moderate and severe dry eye models using three methods in rabbits. BMC Ophthalmol 13:50, 2013

14. Martin MR, Mason CA: The seventh cranial nerve of the rat. Visualization of efferent and afferent pathways by cobalt precipitation. Brain Res 121:21-41,1977

15. Nguyen DH, Toshida H, Schurr J, Beuerman RW: Microarray analysis of the rat lacrimal gland following the loss of parasympathetic control of secretion. Physiol Genomics 17:108-118, 2004

16. Oral E, Aydin MD, Aydin N, Ozcan H, Hacimuftuoglu A, Sipal S, Demirci E: How olfaction disorders can cause depression? The role of habenular degeneration. Neuroscience 240:63-69, 2013

17. Paulsen F: The human nasolacrimal ducts. Adv Anat Embryol Cell Biol 170:1-106, 2003

18. Rezek O, Boldogkoi Z, Tombácz D, Kovágó C, Gerendai I, Palkovits M, Tóth IE: Location of parotid preganglionic neurons in the inferior salivatory nucleus and their relation to the superior salivatory nucleus of rat. Neurosci Lett 440:265269, 2008

19. Riemann R, Pfennigsdorf S, Riemann E, Naumann M: Successful treatment of crocodile tears by injection of botulinum toxin into the lacrimal gland: A case report. Ophthalmology 106:2322-2324, 1999

20. Rocha EM, Alves M, Rios JD, Dartt DA: The aging lacrimal gland: Changes in structure and function. Ocul Surf 6:162174, 2008
21. Scott G, Balsiger H, Kluckman M, Fan J, Gest T: Patterns of innervation of the lacrimal gland with clinical application. Clin Anat 27:1174-1177, 2014

22. Sibony PA, Walcott B, McKeon C, Jakobiec FA: Vasoactive intestinal polypeptide and the innervation of the human lacrimal gland. Arch Ophthalmol 106:1085-1088, 1988

23. Situ P, Simpson TL: Interaction of corneal nociceptive stimulation and lacrimal secretion. Invest Ophthalmol Vis Sci 51:5640-5645, 2010

24. Sullivan DA, Hann LE, Soo CH, Yee L, Edwards JA, Allansmith MR: Neural-immune interrelationship: Effect of optic, sympathetic, temporofacial, or sensory denervation on the secretory immune system of the lacrimal gland. Reg Immunol 3:204-212, 1990-1991

25. Ten Tusscher MP, Klooster J, Baljet B, Van der WF, Vrensen GF: Pre- and post-ganglionic nerve fibres of the pterygopalatine ganglion and their allocation to the eyeball of rats. Brain Res 28:315-323, 1990

26. Thakker MM, Huang J, Possin DE, Ahmadi AJ, Mudumbai R, Orcutt JC, Tarbet KJ, Sires BS: Human orbital sympathetic nerve pathways. Ophthal Plast Reconstr Surg 24:360-366, 2008

27. Thomas PB, Samant DM, Zhu Z, Selvam S, Stevenson D, Wang Y, Song SW, Mircheff, AK, Schechter JE, Yiu SC, Trousdale MD: Long-term topical cyclosporine treatment improves tear production and reduces keratoconjunctivitis in rabbits with induced autoimmune dacryoadenitis. $\mathrm{J}$ Ocul Pharmacol Ther 25:285-292, 2009

28. Toshida H, Nguyen DH, Beuerman RW, Murakami A: Evaluation of novel dry eye model: Preganglionic parasympathetic denervation in rabbit. Invest Ophthalmol Vis Sci 48:44684475, 2007

29. Tubbs RS, Steck DT, Mortazavi MM, Cohen-Gadol AA: The nervus intermedius: A review of its anatomy, function, pathology, and role in neurosurgery. World Neurosurg 79:763767, 2013

30. Yasui T, Karita K, Izumi H, Tamai M: Correlation between vasodilatation and secretion in the lacrimal gland elicited by stimulation of the cornea and facial nerve root of the cat. Invest Ophthalmol Vis Sci 38:2476-2482, 1997

31. Zoukhri D: Effect of inflammation on lacrimal gland function. Exp Eye Res 82:885-898, 2006 